\title{
Polarization study of the pulsars in the globular cluster 47 Tucanae
}

\section{F. Abbate ${ }^{* 1,2}$, A. Possenti ${ }^{2}$, C. Tiburzi $i^{3,4}$, W. van Straten ${ }^{5,6}$, E. Barr ${ }^{3,5}$, A. Ridolfi ${ }^{3}$ and P. Freire ${ }^{3}$}

${ }^{1}$ Dipartimento di Fisica G. Occhialini,Università degli Studi di Milano Bicocca, Piazza della Scienza 3, I-20126 Milano, Italy

${ }^{2}$ INAF - Osservatorio Astronomico di Cagliari, Via della Scienza 5, I-09047 Selargius (CA), Italy.

${ }^{3}$ Max-Planck-Institut für Radioastronomie, Auf dem Hügel 69, D-53121 Bonn, Germany

${ }^{4}$ Fakultät für Physik, Universität Bielefeld, Postfach 100131, D-33501 Bielefeld, Germany

${ }^{5}$ Centre for Astrophysics and Supercomputing, Swinburne University of Technology, Mail H30, PO Box 218, VIC 3122, Australia

${ }^{6}$ Institute for Radio Astronomy and Space Research, Auckland University of Technology, Private Bag 92006, Auckland 1142, New Zealand

E-mail: f.abbate@campus.unimib.it

By investigating the linearly polarized component of a pulsar signal at different radio frequencies it is possible to derive constraints on the parallel component of the magnetic field along the line of sight (los) to the pulsar. In this work we have measured the polarimetric properties of 18 known pulsars in the globular cluster 47 Tucanae and we report for the first time the rotation measure (RM) for 13 of them. Our study led to the discovery of a gradient in the RM values of the pulsars across the cluster. This indicates the presence of significant variations in the magnetic field across the very small angular scales beween the pulsars in 47 Tucanae. We discuss the hypotheses that the magnetic field responsible for the gradient is located inside the globular cluster or in the Galactic disk in the direction of the cluster. However, deeper observations with the MeerKAT and/or the SKA1 radio telescopes are necessary to discriminate among the hypotheses.

MeerKAT Science: On the Pathway to the SKA

25-27 May, 2016

Stellenbosch, South Africa

\footnotetext{
${ }^{*}$ Speaker.
} 


\section{Introduction}

Globular clusters are gravitationally bound spherical stellar systems typically found in the bulge or halo of the galaxies. They are among the oldest and densest stellar systems known: the core can reach stellar densities up to $\sim 10^{6}$ times higher than the typical stellar densities in the Galactic disk. Such extreme characteristics make the central region of a globular cluster a breeding ground for the formation of recycled pulsars, in most cases spinning at millisecond periods.

Besides being interesting per se'[1], the presence of a large family of millisecond pulsars can be exploited to constrain some otherwise elusive physical parameters of the innermost part of a globular cluster. In this respect, the dispersion measure (DM) and the rotation measure (RM) of the pulsars can play a unique role. The DM parametrizes the relative delay between the different frequencies of a broadband signal when it travels through an ionized interstellar medium (ISM). If the ionized ISM is also magnetized, and the propagating signal is linearly polarized, then the signal will undergo Faraday rotation (i.e. a rotation of the polarization position angle). The amount of this effect is quantified by the RM and depends on both the strength of the parallel component of the magnetic field and on the density of the free electrons along the line of sight.

47 Tucanae is the second brightest globular cluster in the night sky and is one of the closest to the Sun. It contains 25 known millisecond pulsars [2] and the total population is estimated to be $\sim 100-200[3,4]$. Almost all the pulsars are located in the central region of the cluster (i.e. within a diameter of $\sim 2$ arcmin) [5, 6, 7]. DM studies performed using the pulsars of 47 Tucanae led to the first detection of ionized gas inside a globular cluster [8]. A parallel study performed on the RM of the pulsars in 47 Tucanae can potentially put constraints on (or reveal for the first time) the presence of a magnetic field inside a globular cluster. Moreover, it could help to study the magnetic turbulence of the ISM on the very small scales associated with the projected positions of the pulsars within the cluster. A similar study has been recently undertaken on the pulsars in the globular cluster Terzan 5 [9].

\section{Results}

The observations were carried out at the Parkes radio telescope over a time span of almost a year from April 2014 to March 2015. The data were obtained in the $20 \mathrm{~cm}$ band, with the central beam of the Multibeam receiver, recorded with the CASPSR backend, and analyzed (including polarization and flux calibration) using the PSRCHIVE software package [10]. The RM values were measured with the PSRCHIVE tool rmfit using the implementation described in [11] and with a self-made code created following the procedure described in Tiburzi et al. [12]. The errors on the values of RM were estimated with a Monte Carlo simulation. Results were considered for subsequent modelling only for the pulsars showing bright enough polarization profiles and having a significant determination of the RM.

The obtained RM values are plotted as a function of DM in Figure 1. These measures are not compatible with a constant RM value across the entire cluster. The scatter in the RM values can be caused either by variations of the magnetic field or of the electron density. The dependance on the electron density can be explored with this plot since both RM and DM relate in the same way to 


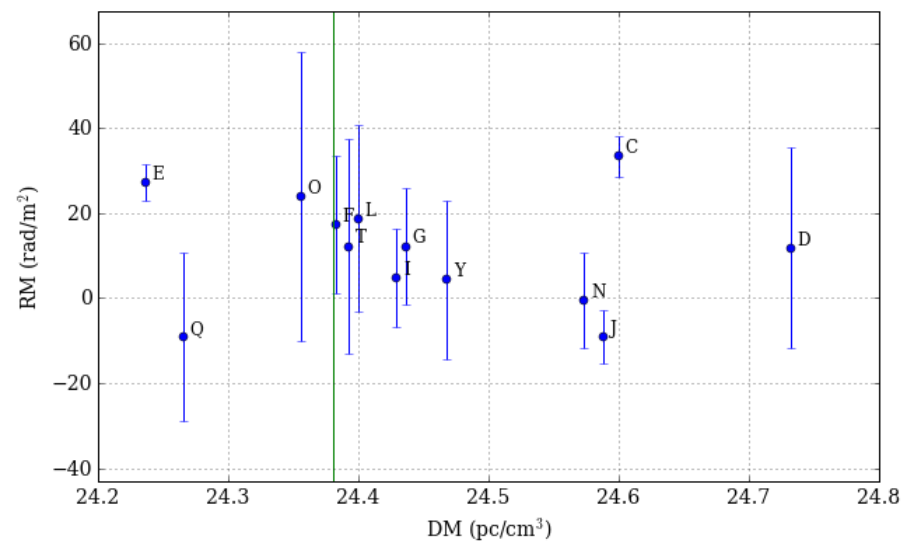

Figure 1: Plot of RM as a function of DM. The green line is at the value of DM at the center of the cluster as measured by Freire et al. [8] . All errors are at two sigma.

it. The absence of any correlation suggests that the variations are most likely caused by a magnetic field which varies across the lines of sight to the various pulsars.

The occurrence of a linear correlation between the RM values and the position of the pulsars relative to the cluster center was investigated with a chi square minimization algorithm. In particular, this algorithm selected the direction along which the correlation was maximal. The angle of the best fit direction is $\sim 60^{\circ}$, measured clockwise from the East direction (positive RA). The correlation along this axis is shown in Figure 2. It has a reduced chi square of 0.725 with 11 degrees of freedom. The gradient is found to be very strong, $0.74 \pm 0.05 \mathrm{rad} \mathrm{m}^{-2} \operatorname{arcsec}^{-1}$. The model reported in Figure 3 shows the intensity and direction of the gradient. This model was constructed assuming the observed gradient was constant throughout the central region of the cluster.

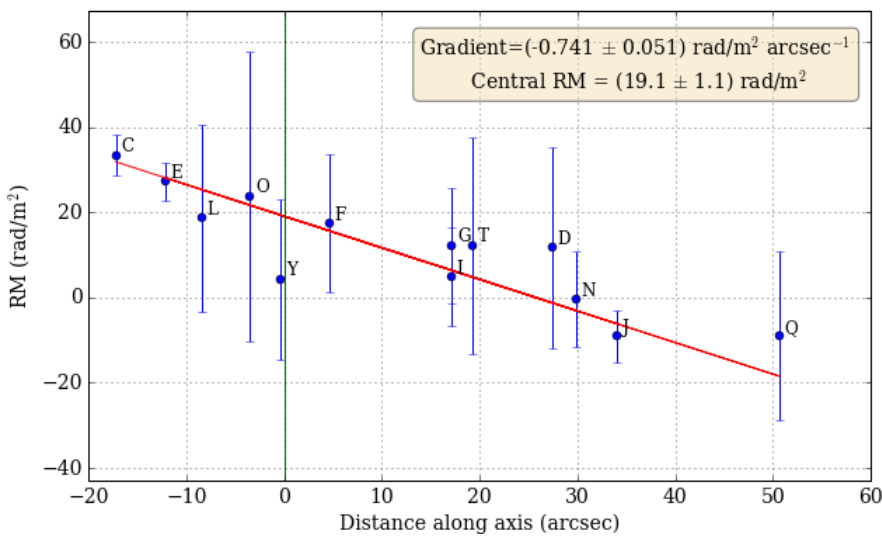

Figure 2: RM as a function of the position of the pulsars in 47 Tucanae projected along the direction that shows the highest correlation (see text). The green line is the position of the optical center of the cluster [13]. All errors are at two sigma. 


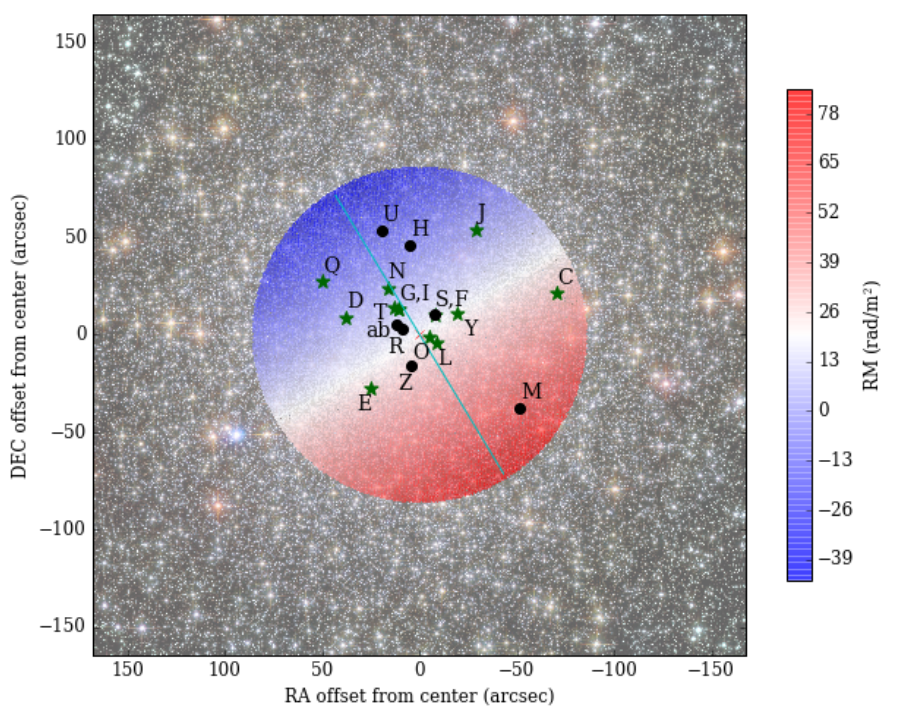

Figure 3: Model of the RM we expect to see if the observed gradient was constant throughout the cluster. The cyan line indicates the direction of the measured gradient. The green stars represent the pulsars with a measured RM, the black dots the ones without a measured RM.

\section{Preliminary discussion}

The results indicate the presence of a magnetic field which is changing across the small angular scales associated with the projected positions of the pulsars in the central region of 47 Tucanae. Preliminary research into the geometry of the observed distribution of the RM values shows the occurrence of a gradient in RM projected across the central part of the cluster. The magnetic field responsible for that could be located in the Galactic disk or inside the cluster. If it resides in the Galactic disk, it could be generated by a magnetic cloud along the line of sight: the required strength of the field within the cloud is not extreme, but the strong gradient suggests a variability in the RM of the ISM that is not detected in the RM maps obtained looking at extragalactic sources at these Galactic latitudes [14]. If, instead, the RM gradient is due to a field located in the central regions of the cluster, it should be very intense, $\sim 200 \mu \mathrm{G}$, assuming a constant field with a toroidal geometry. This corresponds to $\sim 1-2$ orders of magnitude greater than the equipartition value derived from McDonald and Zijlstra [15]. Additional investigations and simulations are ongoing for better constraining the geometry and intensity of the magnetic field affecting the RM values for the pulsars in 47 Tucanae.

Radio telescopes currently under construction in the southern hemisphere, like MeerKAT [16] and SKA could greatly aid in this search. MeerKAT will have, once completed, a collecting area $\sim 3$ times larger than the Parkes radio telescope. This will guarantee, with the same integration time, an increase in sensitivity of $\sim 3$ times. Thanks to this increase and to the larger bandwidth it will be possible to improve the quality of the results and, possibly, obtain RM values for some of the other pulsars of the cluster. With the advent of SKA1-MID the increase in sensitivity achievable with the same integration time is $\sim 10$ times. Observations with these radio telescopes could lead to a decisive proof of the existence of (or lack of) an intrinsic magnetic field in 47 Tucanae. 


\section{Acknowledgements}

With the support of the Ministry of Foreign Affairs and International Cooperation, Directorate General for the Country Promotion (Bilateral Grant Agreement ZA14GR02 - Mapping the Universe on the Pathway to SKA)

\section{References}

[1] F. Camilo and F. A. Rasio, Pulsars in Globular Clusters, ASP Conference Series, Vol 328, p. 147, 2005.

[2] Z. Pan et al., Discovery of two new pulsars in 47 Tucanae (NGC 104), Monthly Notices of the Royal Astronomical Society: Letters, 426:L26- L30, 2016.

[3] F. Camilo et al., Observations of 20 millisecond pulsars in 47 tucanae at 20 centimeters, The Astrophysical Journal, 535:975-990, 2000.

[4] P. J. Turk and D. R. Lorimer, An empirical Bayesian analysis applied to the globular cluster pulsar population, Monthly Notices of the Royal Astronomical Society, 436:3720-3726, 2013.

[5] P. C. C. Freire et al., Further results from the timing of the millisecond pulsars in 47 Tucanae, Monthly Notice of the Royal Astronomical Society, Volume 340, Issue 4, pp. 1359-1374.

[6] A. Ridolfi et al., Long-term observations of the pulsars in 47 Tucanae - I. A study of four elusive binary systems, Monthly Notices of the Royal Astronomical Society, Volume 462, Issue 3, p.2918-2933

[7] P. C. C. Freire et al., Long-term observations of the pulsars in 47 Tucanae - II. Proper motions, accelerations and jerks, eprint arXiv: 1706.04908

[8] P. C. C. Freire et al., Detection of ionized gas in the globular cluster 47 tucanae, The Astrophysical Journal Letters, 557:L105-108, 2001.

[9] A. Ho et al., Rotation measures of globular cluster pulsars as a unique probe of the galactic magnetic field, American Astronomical Society, AAS Meeting n.223, id.153.18, 2013.

[10] A. W. Hotan, W. van Straten and R. N. Manchester, PSRCHIVE and PSRFITS: An Open Approach to Radio Pulsar Data Storage and Analysis, Publications of the Astronomical Society of Australia, Volume 21, Issue 3, pp. 302-309.

[11] J. L. Han, R. N. Manchester, A. G. Lyne, G. J. Qiao and W. van Straten, Pulsar Rotation Measures and the Large-Scale Structure of the Galactic Magnetic Field, The Astrophysical Journal, Volume 642, Issue 2, pp. 868-881.

[12] C. Tiburzi et al. The high time resolution universe survey - ix. polarimetry of long-period pulsars, Monthly Notices of the Royal Astronomical Society, 436:3557-3572, 2013.

[13] D. E. McLaughlin et al., Hubble space telescope proper motions and stellar dynamics in the core of the globular cluster 47 tucanae, The Astrophysical Journal Supplement Series, 166:249-297, 2006.

[14] N. Oppermann et al., An improved map of the galactic faraday sky, Astronomy and Astrophysics, 575, 2015.

[15] I. McDonald and A. A. Zijlstra, Globular cluster interstellar media: ionized and ejected by white dwarfs, Monthly Notices of the Royal Astronomical Society, 446:2226-2242, 2015.

[16] R. S. Booth and J. L. Jonas, An overview of the Meerkat project, African Skies, 16:101, 2012. 